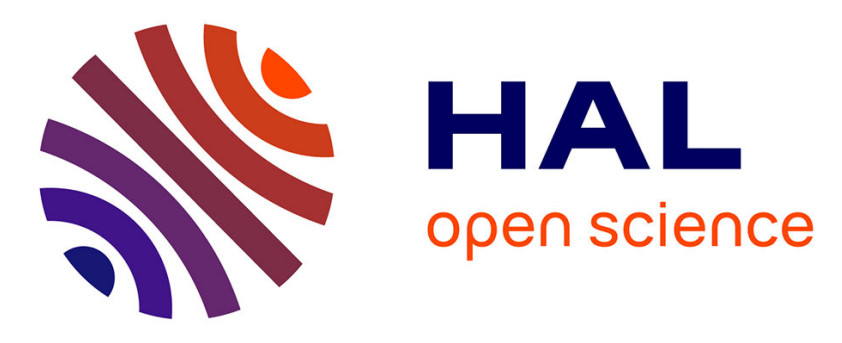

\title{
Impact of biofilm-induced heterogeneities on solute transport in porous media
}

\author{
T. Kone, F. Golfier, L. Orgogozo, C. Oltean, E. Lefèvre, J. C. Block, M. A. \\ Bues
}

\section{- To cite this version:}

T. Kone, F. Golfier, L. Orgogozo, C. Oltean, E. Lefèvre, et al.. Impact of biofilm-induced heterogeneities on solute transport in porous media. Water Resources Research, 2014, 50 (11), pp.9103-9119. 10.1002/2013WR015213 . hal-01301700

\section{HAL Id: hal-01301700 \\ https://hal.science/hal-01301700}

Submitted on 8 Jul 2021

HAL is a multi-disciplinary open access archive for the deposit and dissemination of scientific research documents, whether they are published or not. The documents may come from teaching and research institutions in France or abroad, or from public or private research centers.
L'archive ouverte pluridisciplinaire $\mathbf{H A L}$, est destinée au dépôt et à la diffusion de documents scientifiques de niveau recherche, publiés ou non, émanant des établissements d'enseignement et de recherche français ou étrangers, des laboratoires publics ou privés.

$$
\text { Copyright }
$$




\section{Water Resources Research}

\section{RESEARCH ARTICLE Impact of biofilm-induced heterogeneities on solute transport \\ 10.1002/2013WR015213 in porous media}

Key Points:

- Biofilm thickness was characterized

by tracer experiments with Blue

Dextran

- Biofilm growth induces

heterogeneities influencing transport

in porous media

- An experimental correlation was

found between porosity and

dispersivity

Correspondence to:

F. Golfier,

fabrice.golfier@univ-lorraine.fr

Citation:

Kone, T., F. Golfier, L. Orgogozo,

C. Oltéan, E. Lefèvre, J. C. Block, and

M. A. Buès (2014), Impact of biofilm-

induced heterogeneities on solute

transport in porous media, Water

Resour. Res., 50, 9103-9119,

doi:10.1002/2013WR015213.

Received 22 DEC 2013

Accepted 22 OCT 2014

Accepted article online 28 OCT 2014

Published online 26 NOV 2014

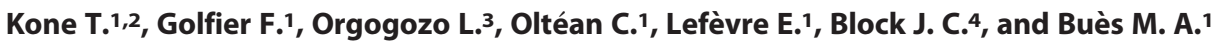 \\ ${ }^{1}$ Université de Lorraine, CNRS, CREGU, GeoRessources Laboratory, Nancy, France, ${ }^{2}$ Université Nangui Abrogoua UFR Sciences \\ et Gestion de I'Environnement, Abidjan, Côte d'Ivoire, ${ }^{3}$ Géosciences Environnement Toulouse, Observatoire de Midi- \\ Pyrénées, Université de Toulouse, Toulouse, France, ${ }^{4}$ Université de Lorraine and CNRS, LCPME UMR 7564, Nancy, France
}

\begin{abstract}
In subsurface systems, biofilm may degrade organic or organometallic pollutants contributing to natural attenuation and soil bioremediation techniques. This increase of microbial activity leads to change the hydrodynamic properties of aquifers. The purpose of this work was to investigate the influence of biofilm-induced heterogeneities on solute transport in porous media and more specifically on dispersivity. We pursued this goal by (i) monitoring both spatial concentration fields and solute breakthrough curves from conservative tracer experiments in a biofilm-supporting porous medium, (ii) characterizing in situ the changes in biovolume and visualizing the dynamics of the biological material at the mesoscale. A series of experiments was carried out in a flow cell system $\left(60 \mathrm{~cm}^{3}\right)$ with a silica sand $(\Phi=50-70$ mesh) as solid carrier and Shewanella oneidensis MR-1 as bacterial strain. Biofilm growth was monitored by image acquisition with a digital camera. The biofilm volume fraction was estimated through tracer experiments with the Blue Dextran macromolecule as in size-exclusion chromatography, leading to a fair picture of the biocolonization within the flow cell. Biofilm growth was achieved in the whole flow cell in 29 days and up to $50 \%$ of void space volume was plugged. The influence of biofilm maturation on porous medium transport properties was evaluated from tracer experiments using Brilliant Blue FCF. An experimental correlation was found between effective (i.e., nonbiocolonized) porosity and biofilm-affected dispersivity. Comparison with values given by the theoretical model of Taylor and Jaffé (1990b) yields a fair agreement.
\end{abstract}

\section{Introduction}

In groundwater aquifers, biofilm may degrade organic or organometallic pollutants contributing to natural attenuation and soil bioremediation techniques. Biofilms are sessile communities of microorganisms that are attached to liquid or solid interfaces, e.g., the pore walls of a water-saturated aquifer, and embedded within a matrix of extracellular polymeric substances (EPS) that they have produced [Donlan and Costerton, 2002]. In flow systems such as lab columns or aquifers, biofilm growth is related to nutrient availability which itself depends on nutrient concentration within the fluid phase and on the hydrodynamics. Investigating the complex coupling between flow and microbial growth processes remains a major challenge for studies of solute transport in biofilm-coated porous media. Due to the biological activity (growth and metabolism), hydrodynamic related parameters in porous media may be impacted. As biofilm grows, indeed, porosity and permeability decrease whereas the longitudinal and transverse dispersivities increase. If we focus hereafter on the impact due to biomass accumulation, note that microbial production of gases [Sanchez de Lozada et al., 1994] or biologically driven mineral precipitation [De Muynck et al., 2010] may be other possible causes of clogging [Baveye et al., 1998]. This phenomenon leads to significant issues both in environmental or engineering related applications, which include clogging of landfill leachate collection system [VanGulck and Rowe, 2004] and impact of the biomass distribution on contaminant fate in groundwater [Bielefeldt et al., 2002a, 2002b] or on remediation strategies [Sturman et al., 1995]. Conversely, beneficial aspects may be also gained from such a biological plugging. The clogging of low-permeability zones, for instance, may enhance oil recovery or improve wastewater quality for aquifer recharge [MacLeod et al., 1988]. Charbonneau et al. [2006] suggest that biofilm in fractures may prevent or slow down the contaminant propagation in fractured subsurface formation. In a similar way, other authors [Ebigbo et al., 2010] have proposed to biologically enhance subsurface sequestration of supercritical $\mathrm{CO}_{2}$ by reducing permeability of the caprock. 
Historically, a special focus has been put on the decrease of hydraulic conductivity with biofilm growthrelated to the reduction in pore interconnectivity-that has been intensively observed [Mitchell and Nevo, 1964; Torbati et al., 1986; MacLeod et al., 1988; Taylor and Jaffé, 1990a; Cunningham et al., 1991; Vandevivere and Baveye, 1992a; Kim and Fogler, 2000; Thullner et al., 2002a, 2002b; Bielefeldt et al., 2002a, 2002b; Seifert and Engesgaard, 2007; Karrabi et al., 2011]. This decrease in permeability caused by bioclogging, is due to accumulation of biofilm materials in pores (that is to say microorganisms and polymeric substances). Torbati et al. [1986] observed that biofilm plugged mainly the larger pores whereas, according to Nambi et al. [2003], biomass development is preferentially dominated by shear stress rate. Impact of morphological structure of the biofilm accumulation has also been questioned [Paulsen et al., 1997; Dupin and McCarty, 2000]. Abundance of EPS depends on microbial strain nature and physiology but they were found to drastically reduce permeability even at low concentration of EPS in porous medium if spatially distributed [Mitchell and Nevo, 1964; Baveye et al., 1998; Vandevivere and Baveye, 1992b; Thullner et al., 2002a, 2002b]. For high concentrations, formation of highly conductive channels within the porous medium has been observed [Stewart and Fogler, 2001]. Thus, beyond the biofilm density (biofilm thickness), it is the spatial distribution of biofilms and their morphology (sparse or continuous biofilm, filament-type biofilm) that influence porous medium permeability [Paulsen et al., 1997; Nambi et al., 2003; Vayenas et al., 2002].

This spatial distribution of biofilm materials naturally impacts not only flow mechanisms but also the whole transport process of solutes. More recently, dispersion was directly correlated with the biofilm extent in porous medium [Taylor et al., 1990; Sharp et al., 1999; Engesgaard et al., 2006; Seifert and Engesgaard, 2007, Yang et al., 2013]. The impact of biofilm growth on dispersion was visually shown through flow channelization in several studies [Kildsgaard and Engesgaard, 2002; Oates et al., 2005; Sharp et al., 2005; Rockhold et al., 2005; Seymour et al., 2007] due to bioclogging [Thullner et al., 2002a; Seifert and Engesgaard, 2007]. Dispersion increases up to 2 orders of magnitude for certain experiments [Taylor et al., 1990; Bielefeldt et al., 2002a]. Here again biofilm spatial distribution more than biofilm density plays a crucial role on dispersive properties of porous media. Results of column experiments reported by Bielefeldt et al. [2002a] support this assumption. They observed an increase in dispersion for earlier stages of biofilm growth before decreasing with biofilm maturation. Following the authors, this behavior results from the heterogeneous distribution of biofilm forming bacteria during the early stage of development, which becomes more uniform with time.

To summarize, biofilm-induced heterogeneities impact solute transport at two scales: (i) at the pore scale where the existence of a fluid/biofilm interface leads to different characteristic times of transport in both phases, i.e., advection and diffusion in fluid phase on one hand and diffusion in biofilm phase (if channelization is neglected in biofilm structure) on the other hand [Orgogozo et al., 2013]; (ii) at the Darcy scale (i.e., mesoscale or core scale) where nonuniform biofilm development creates preferential flow paths. Better understanding on this issue-crucial if we wish to drive in situ bioremediation techniques toward a better control of permeability and mass transport properties-has been gained from the above mentioned studies. Nonetheless, two major criticisms should be raised. First of all, experimental monitoring for most of the studies was performed in columns and hence, limited to solute breakthrough curves. An improved insight on in situ biomass distribution has come from advances in microbiology methods (genetically marked or bioluminescent bacteria) and noninvasive observation techniques: light transmission method [e.g., Yarwood et al., 2002] for 2-D porous media and microscopy-based method [Leis et al., 2005], magnetic resonance microscopy [Seymour et al., 2004a, 2004b, 2007], or X-ray tomography in 3-D [Iltis et al., 2011; Davit et al., 2011]. Unfortunately, most of them were limited to postmortem observations or remained purely qualitative [Iltis et al., 2011; Davit et al., 2011]. Very few studies succeeded to acquire change of spatial concentration fields in a biofilm-coated porous medium over time [Yarwood et al., 2002; Rockhold et al., 2005; Seifert and Engesgaard, 2012]. Second, and above all, biofilm related parameters (e.g., biofilm thickness, volume fraction, EPS, and/or bacteria concentration) were usually obtained postmortem, i.e., through destructive techniques. In particular, the biofilm volume fraction or biovolume, denoted by $\varepsilon_{\omega}$, which is a key parameter to investigate and analyze the complex coupling involved with fluid flow and mass transport processes, is practically never measured or at least, not locally. Several studies [Taylor and Jaffé, 1990a, 1990c; Cunningham et al., 1991] have related changes in porosity to measurement of biofilm thickness although this parameter is highly space dependent (biofilm heterogeneity) and requires destructive sampling of the porous material. VanGulck and Rowe [2004] have disassembled their experimental column to measure the drainable porosity within each section. Other authors have used measures of permeability reduction to 
estimate approximately the changes in porosity from comparison with numerical simulations [Dupin and McCarty, 2000; Thullner et al., 2002a; Charbonneau et al., 2006] or from empirical relations relating both parameters [Seifert and Engesgaard, 2007]. Cunningham et al. [1991] and Bielefeldt et al. [2002a] have computed the average effective porosity of the column from breakthrough curves but failed to distinguish the combined retardation effect of the tracer due to diffusion and adsorption into the biofilm. These effects of diffusional loss of tracer into the biomass were evaluated further by Seifert and Engesgaard [2007] using a constant retardation factor. More recently, Seifert and Engesgaard [2012] used this method to correct measurement of the average porosity of a 2-D porous medium from dye tracer injections.

The purpose of this work is to investigate the influence of biofilm-induced heterogeneities on solute transport in porous media with a particular focus on changes in longitudinal dispersivity. The originality of this study is twofold: (i) to monitor both spatial concentration fields and determine solute breakthrough curves through conservative tracer experiments within a flow cell at different stages of biofilm growth, (ii) to propose a relevant technique for in situ biovolume characterization and visualize the dynamics of the microbial biofilm at the mesoscale. This latter method is based on tracer experiments with the Blue Dextran macromolecule, which has already been employed to evaluate time residence distribution in submerged filters [Jiménez et al., 1988] or for ex situ determination of biovolume in batch experiments [Di laconi et al., 2004] but was never used for in situ measurement of biofilm volume fraction in porous media to the best of our knowledge. Combination of these porosity measures with changes in dispersivity over time provides a unique insight on interactions between biofilm growth and flow and transport processes in porous media.

\section{Materials and Methods}

\subsection{Porous Medium}

A 2-D glass flow cell of dimensions $10 \mathrm{~cm} \times 10 \mathrm{~cm}$ and thickness $0.6 \mathrm{~cm}$ has been used for the experiment. It was packed with a homogeneous porous material made of silica sand (CAS Number 14808-60-7, SigmaAldrich) with particle size 50-70 mesh (210-297 $\mu \mathrm{m}$ of grain diameter). The grain size in the range of fine to medium sand is typical of alluvial aquifer material. This type of carrier was found to be suitable for supporting biofilm growth and showed no significant adsorption of the dyes (Brilliant Blue FCF and Blue Dextran).

A constant sand mass of $78.32 \mathrm{~g}$ was retained during the filling procedure. The initial porosity $\varepsilon$ after packing was measured by volume balance and a value of $0.40 \pm 0.01$ was obtained. A series of tracer injection experiments was conducted later (cf. section 2.6.) and comparison with numerical simulations led to the same estimate $(\varepsilon=0.40)$. The hydraulic conductivity of the packed bed was also determined and was about $4 \times 10^{-4} \mathrm{~m} / \mathrm{s}$.

\subsection{Experimental System}

The experimental setup is illustrated in Figure 1. It was designed for both biofilm cultivation and tracer injection experiment. The flow cell has a mixing reservoir to ensure a uniform injection and prevent bioplugging of the inlet or channeling effect due to injection ports (Figure 2). A gear pump (Ismatec, MCPZ Process IP 65) equipped with a magnetic head (Micropump, P/N 820921006 model O/C GAX21.CFSB) drives fluid through the experimental cell to a waste container. A bubble trap is used to limit air bubbles passing through the flow cell. These individual components are connected by sterile C-Flex tubing (internal diameter: 0.125 mm, Cole-Palmer, 6424-67), valves, and different triple-inlet connectors (PP/KYNAR LUER, 88084). After packing, flow cell and tubings are initially autoclaved at $121^{\circ} \mathrm{C}$ for $15 \mathrm{~min}$. Valve manifolds are sterilized chemically with biocides (Amphospray 41, Anios) and rinsed with sterile deionized water. Fluid tanks and stock solutions are also autoclaved prior to use. Experiments are operated in a temperature-controlled chamber $\left(30^{\circ} \mathrm{C}\right)$ during biofilm growth.

First, carbon dioxide $\left(\mathrm{CO}_{2}\right)$ was flushed into the porous medium. A gas filter $(\varnothing=0.22 \mu \mathrm{m})$ was connected to the flow cell inlet for filtering the incoming $\mathrm{CO}_{2}$ and prevent any microbial contamination. Saturating the flow cell with $\mathrm{CO}_{2}$ avoids air bubbles trapped in the porous medium when saturation with water begins. Next, the flow cell is slowly purged with $0.9 \% \mathrm{NaCl}$ solution of sterilized water to avoid bacterial cell lysis until complete saturation.

\subsection{Bacterial Culture}

Shewanella oneidensis MR-1 an aerobic/facultative anaerobic gram-negative bacterial strain was used to grow biofilms. It was initially grown on agar plates prepared with $25 \mathrm{~g} \mathrm{~L}^{-1}$ of Luria Broth-Miller and $15 \mathrm{~g} \mathrm{~L}^{-1}$ of agar 


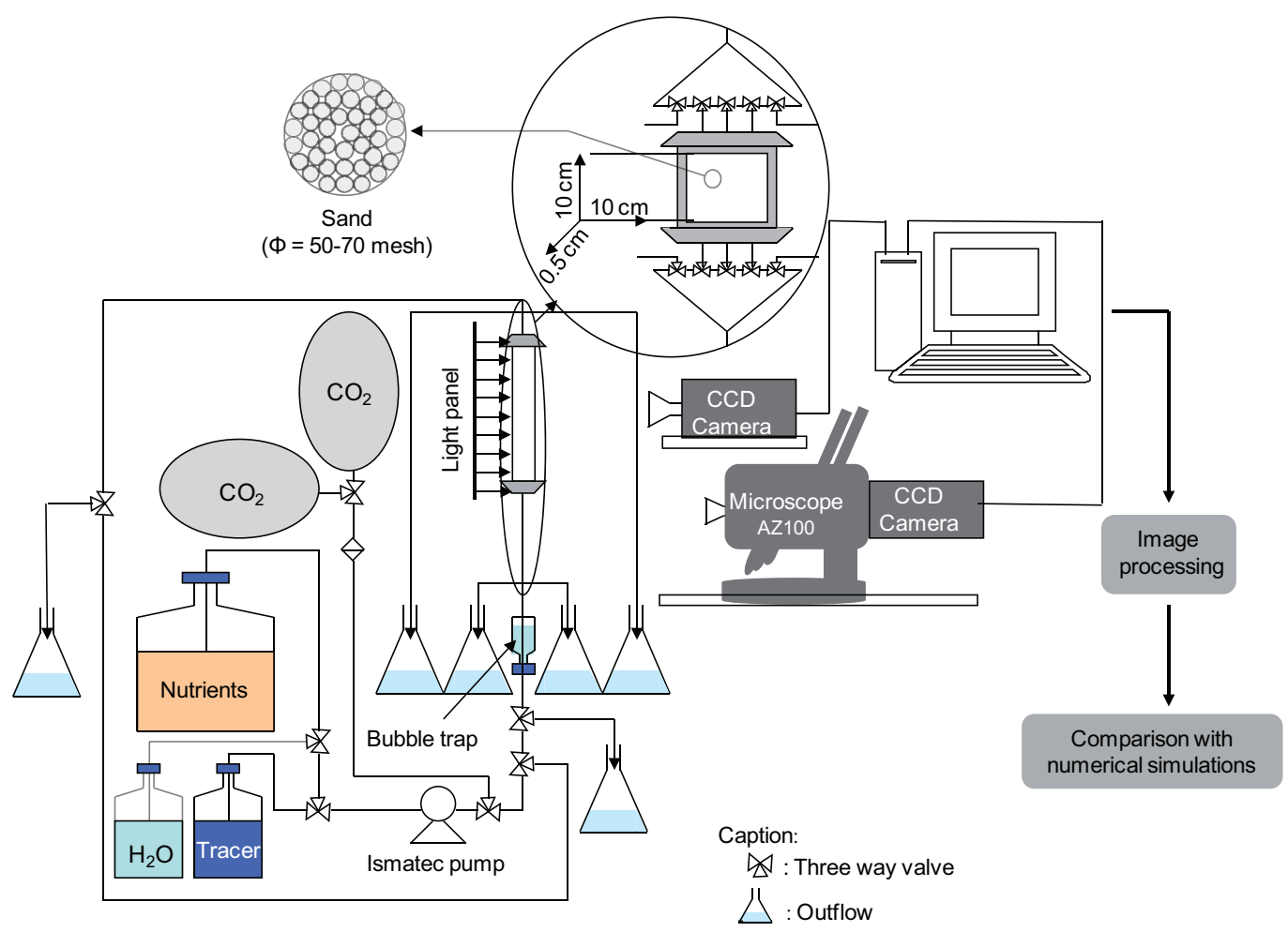

Figure 1. Experimental setup.

(BD Difco). A startup bacterial suspension was prepared with a single colony from agar plates. The colony was inoculated into LML +F medium (LML solution supplemented with fumarate as electron acceptor) at $\mathrm{pH} 7.4$ and at $30^{\circ} \mathrm{C}$ with shaking at $250 \mathrm{rpm}$ [Teal et al., 2006]. Then $400 \mathrm{~mL}$ inoculum was prepared. For this purpose, four sterile flasks of $250 \mathrm{~mL}$ each, containing $100 \mathrm{~mL}$ of $\mathrm{LML}+\mathrm{F}$ were replicated with the previous bacterial suspension and incubated at $30^{\circ} \mathrm{C}$ with shaking at $160 \mathrm{rpm}$. The incubation was stopped after approximately 3-4 $\mathrm{h}$ when the optical density at $600 \mathrm{~nm}\left(\mathrm{OD}_{600}\right)$ reaches 0.2 , which corresponds to a bacterial concentration of $\sim 10^{8}$ bacteria $\mathrm{mL}^{-1}$. Bacterial suspensions in the four flasks were next transferred under sterile conditions into a $1 \mathrm{~L}$ sterile flask equipped with flow tubes.

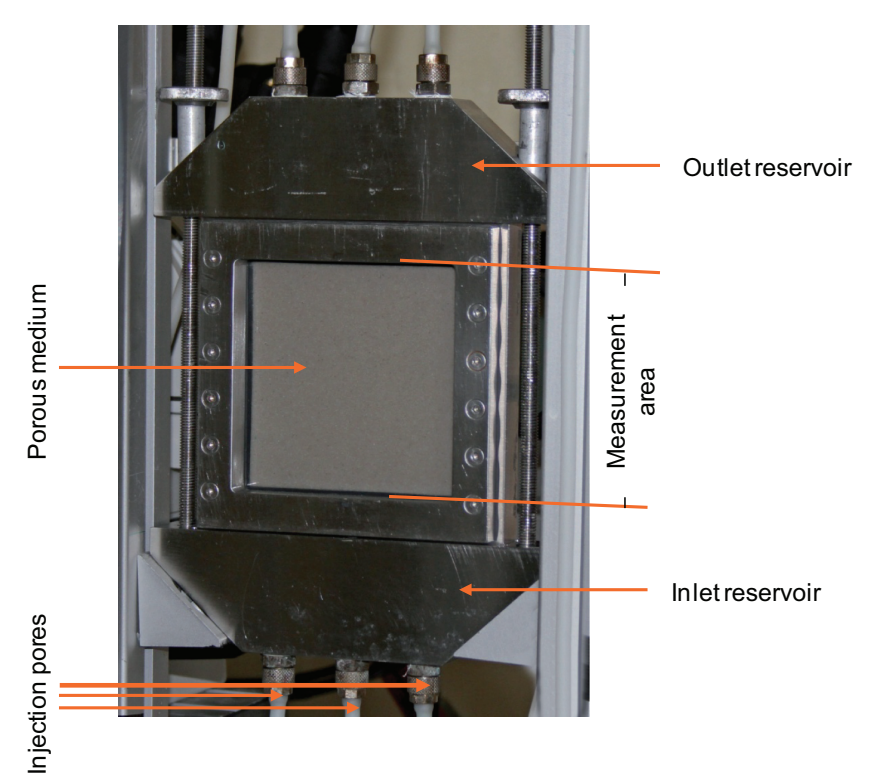

Figure 2. Flow cell features.

\subsection{Biofilm Formation}

To allow the biofilm formation in the flow cell filled with porous medium, the $1 \mathrm{~L}$ flask containing the bacterial suspension was connected to the flow system under aseptic conditions, and bacterial suspension was circulated until five pore volumes of inoculum went through the porous medium. This ensured that the whole porous medium was saturated by bacterial suspension. Then, the system was let at rest for at least $1 \mathrm{~h}$. Finally, Luria Broth-Miller (1.5 g/L) nutritive broth was used as electron donor and was supplemented with 20 $\mathrm{mmol} / \mathrm{L}$ of fumarate as electron acceptor. This solution was continuously supplied with the pump. The 
flow cell system was operated for 29 days under a continuous flow rate of nutrient $\left(Q=8 \times 10^{-2} \mathrm{~cm}^{3} \mathrm{~s}^{-1}\right)$. The flow rate was chosen to generate high shear stress throughout biofilm growth so that bacterial detachment was minimized during tracer tests. Changes in flow cell transparency was checked at regular intervals through the measurements of the blank absorbance (i.e., the absorbance of the flow cell saturated with $0.9 \% \mathrm{NaCl}$ solution). No local decrease of gray-level intensity (i.e., no local increase of transparency) was ever observed that could indicate any significant biomass detachment.

\subsection{Tracer Injection Experiment and Image Processing}

At $t=0,17$, and 29 days, conservative tracer injections were carried out for characterizing both dispersivity and biovolume within the flow cell. Visible light transmission method was used for these measures. A Princeton Instruments $®$ PI-MAX3 (16 Bits, $1024 \times 1024$ pixels) digital camera, aimed perpendicular to the cell, was employed throughout the experiments to follow spatial and temporal changes of tracer concentration within the porous medium. This intensified Couple Charge Device (CCD) camera was controlled with a Princeton Instruments ${ }^{\circledR}$ WinSpec/32, Version 2.5.25.2 software. Since a dyed tracer was used, the CCD camera lens was supplemented with a band-pass filter 600-650 nm (Laser Components SAS®, Type 628BP50 SHO/128571 G.F). The transparent cell was back lighted with a LED light source during images capture. Note that a peculiar attention was given to the backlighting system so that a homogeneous and constant light intensity was provided during all the experiment. Two different conservative tracers (Brilliant Blue FCF and Blue Dextran prepared with $0.9 \% \mathrm{NaCl}$ solution) have been used for biovolume and dispersivity measurement. It should be emphasized that Brillant Blue was used as an ideal pollutant to explore the impact of the biofilm phase on the fate of a miscible contaminant plume. On the contrary, Blue Dextran was only used to investigate and quantify the presence of biomass within the flow cell, i.e., the biofilm volume fraction which was a crucial parameter for interpreting our experiments. It could not have been considered as representative of the behavior of a classical pollutant due to its high molecular weight. Before tracer injection experiments were performed, a calibration was required for converting raw images of light intensity obtained with the camera into concentration field using Beer-Lambert law. If this procedure is classical, additional complexity stems from the changes induced by biofilm growth on the light absorption properties of the porous medium (the light intensity of raw images of the porous material decreases of up to 40,000 gray levels between $t=0$ and $t=29$ days). In other words, a pixel-by-pixel calibration is required for each tracer and before each injection experiment (i.e., at $t=0,17$, and 29 days) for correcting intensity variation due to biofilm and light source inhomogeneity. A separate linear calibration curve for each pixel from the $C C D$ camera is thus obtained by measuring the light intensity for a series of standard solutions of known concentration. Calibration and injection experiment have been conducted for both tracer over a 2 day period but this lapse of time between dispersivity and effective porosity measurement was assumed negligible when interpreting data.

First series of tracer injection experiments were performed with Brilliant blue FCF (Acros Organics, CAS Number: $3844-45-9, \lambda_{\max }=625 \mathrm{~nm}$ ) as conservative tracer. Two different injection flow rates have been considered, respectively, $Q=1.33 \times 10^{-2} \mathrm{~cm}^{3} \mathrm{~s}^{-1}$ and $Q=8 \times 10^{-2} \mathrm{~cm}^{3} \mathrm{~s}^{-1}\left(v=2.2 \times 10^{-3} \mathrm{~cm} \mathrm{~s}^{-1}\right.$ and $v=1.33$ $\times 10^{-2} \mathrm{~cm} \mathrm{~s}^{-1}$ for the corresponding flow velocities), in order to investigate changes in dispersivity with pore water velocity $v_{\gamma}$ and biofilm growth. Note that these two parameters are not independent since pore water velocity increases over time due to bioplugging (for the same flow rate). For each flow rate, 420 images were automatically captured at regular time interval. The time step was chosen so that five pore volumes of dye solution pass through the porous medium. This volume of solution was found to be sufficient to saturate the entire flow cell with the dye. Before each tracer experiment, the porous medium was washed with $0.9 \% \mathrm{NaCl}$ solution in order to get a blank as similar as possible to the calibration one. Each series of 420 raw RGB images was processed with a Fortran 90 computer code, and gray-level matrix $(1024 \times 1024$ pixels) has been converted into 2-D concentration field. Finally, breakthrough elution curves were reconstructed from these data fields at the outlet (i.e., $x=9 \mathrm{~cm}$ ). Note that effluent measurements could not be carried out for comparison due to the mixing within the outlet reservoir (presence of dead zones). However, mass balance was checked against imaging results and a very good agreement was obtained ( $98 \%$ of the total mass recovered during injection tests).

Two specific issues were faced during the experiments in biotic conditions and need to be mentioned. First, a constant concentration at the inlet of the flow cell cannot be ensured during tracer injection. Indeed, a complete mixing within the inlet reservoir (Figure 2) is essential to this purpose. Although several pore 
volumes of the dye solution have been flushed through the inlet reservoir and homogeneity of dye concentration was checked by absorbance measurement with spectrophotometer at a wavelength of $625 \mathrm{~nm}$, a ramp effect has persisted at the inlet of the system. This experimental artifact needed to be considered during data analysis. Second, the impact of high biocolonization on pixel-by-pixel calibration curves led to light intensity variation of several thousands of gray levels over a few decades of pixels. The nonlinear impact of the biofilm growth on the attenuation coefficient of the biocolonized material for a given pixel made impossible to relate explicitly the decrease in intensity to the volume fraction of biomass. Moreover, this overall decrease of medium transparency combined with strong pixel-to-pixel fluctuations of optical density yielded a quality decrease (low regression coefficient values) of calibration curves for certain pixels as biofilm gets more and more mature. To minimize this experimental bias, images have been postprocessed using a spatial-smoothing algorithm with a running window low-pass filter. A 16-pixel window was found to be the best compromise to assure low noise and reduce loss of information.

\subsection{Biofilm Monitoring}

Complementary information has been collected to provide a complete and accurate picture of biofilm distribution within the flow cell. First, qualitative measurements were carried out by capturing raw images of the flow cell with the CCD camera. By image differencing with the initial snapshot of the porous medium, changes in transparency are exhibited and indicate whether biofilm is present and the zones of preferential growth. Local images of biofilm-coated grains were also captured with the AZ100 Multizoom Microscope (Nikon Instruments Inc.).

In addition to these measurements, an original method to quantify biofilm volume fraction in situ has been used. The objective is to determine directly the effective average porosity from the breakthrough curves between two successive cross sections of the flow cell. This approach has been already employed by Cunningham et al. [1991], Bielefeldt et al. [2002a], and Seifert and Engesgaard [2012], but the use of "classical" miscible tracers (Bromide or bromothymol blue dye) has biased their analysis. The implicit assertion, indeed, that the tracer injected does not penetrate within the biofilm but flows only through the mobile phase (bulk pores) has no real physical justification. The originality of our approach lies in the use of a macromolecule, i.e., Blue Dextran (CAS Number: 87915-386, Sigma-Aldrich), a high molecular weight anionic compound ( $\mathrm{Mw}=2 \times 10^{6} \mathrm{~g} / \mathrm{mol}$ ) classically used in the determination of the void volume for gel-filtration chromatography columns (e.g., in Persson et al. [2006]) and assumed by analogy not to adsorb nor to diffuse into the biofilm phase. Additional information about this dye and about the assumptions related to our measurement method can be found in Appendix A.

For each stage of biofilm growth, tracer experiments were carried out both with Brilliant blue FCF (already described in upper sections) and Blue Dextran. Biovolume measures are carried out as follows. Tracer injection experiment is performed with the Blue Dextran dye and breakthrough curves are calculated at different cross sections, each $1 \mathrm{~cm}$ apart from the other (between $x=2$ and $x=9 \mathrm{~cm}$ ). Calibration was previously done as detailed in section 2.5. Then, the velocity of the center of mass of these elution curves is determined using the classical definition of residence time for a step injection [Luckner and Reissig, 1979] and the average effective porosity $\varepsilon_{\gamma}(x)$ between two successive cross sections is given by:

$$
\varepsilon_{\gamma}(x)=\frac{Q}{S \bar{v}_{\gamma}(x)}
$$

where $S$ is the flow section area $\left(6 \mathrm{~cm}^{2}\right)$ and $\bar{v}_{\gamma}(x)$ the cross-section averaged pore water velocity in the $x$ coordinate direction. Finally, biovolume $\varepsilon_{\omega}(x)$ and effective porosity $\varepsilon_{\gamma}(x)$ are related by the following relationship:

$$
\varepsilon_{\omega}(x)=\varepsilon-\varepsilon_{\gamma}(x)
$$

with $\varepsilon$ the initial porosity of the flow cell $(\varepsilon=0.40)$.

\section{Results and Discussion}

\subsection{Biofilm Monitoring}

In order to follow the porous medium colonization by the biofilm, raw images were collected up to 29 days. As shown on Figure 3, which summarizes some of the relevant growth periods, the porous medium 


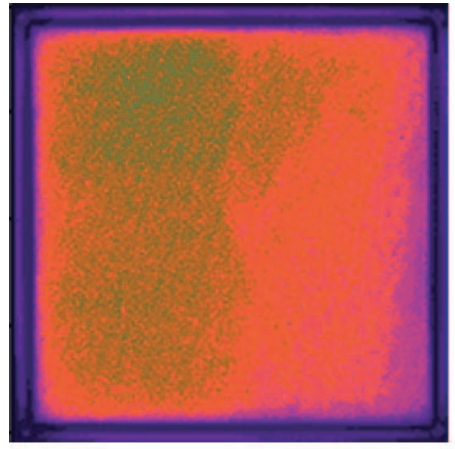

a) $t=0$ (inoculation stage)

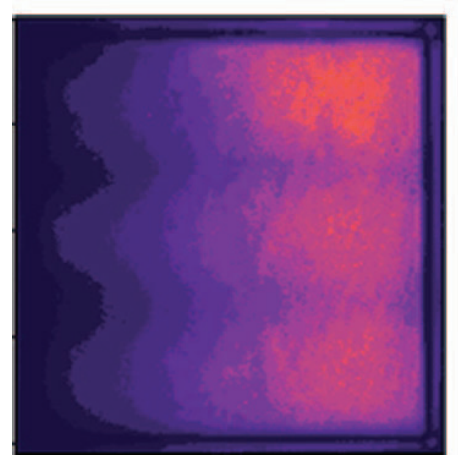

c) $t=17$ days

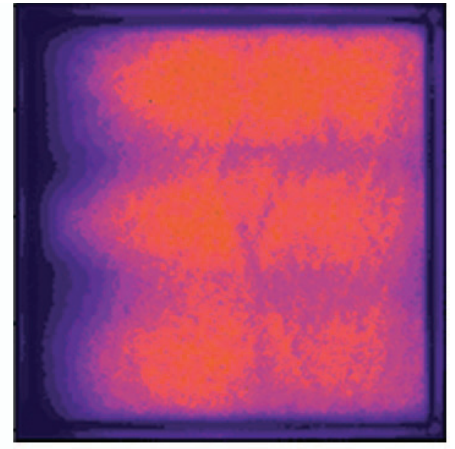

b) $t=10$ days

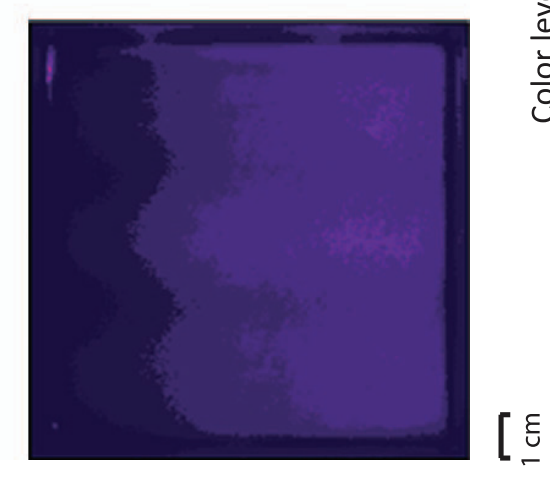

d) $t=29$ days

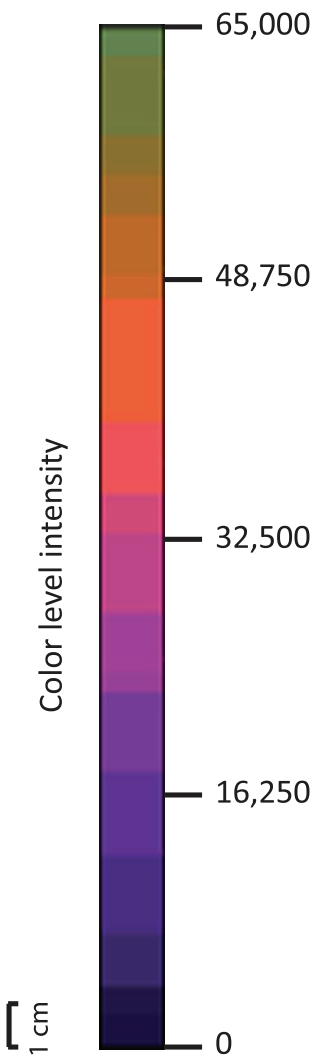

Flow direction

Figure 3. CCD camera raw images showing biofilm growth evolution in porous medium versus time.

transparency decreases as biofilm thickens throughout the experiment. At the end of the experiment (image d, Figure 3), the significant decay in gray-level intensity (at least $-10,000$ and down to $-40,000$ from the initial gray level value of the CCD, that is 65,000 ) indicates that a total coverage of the porous medium was achieved at day 29 , even if stratification exists. Areas close to nutrients source (i.e., the first third of the porous medium from the bottom of the flow cell) are less transparent (low gray level) than top areas. Similarly, biomass development is characterized by a wavy front of propagation close to the inlet (image $b$ and $c$ in Figure 3). Position of low-intensity zones coincides with the location of the three injection ports connected to the upstream of the mixing reservoir. These observations suggest that the injection is not perfectly uniform and high and low shear stress zones subsist. However, it should be noted that these wavy biofilm layers tend "to homogenize" as the biomass front propagates inside the porous medium (image d, Figure 3). If the results of observations from microscope after 29 days (Figure 4) do not allow to clearly show the biofilm distribution, it is apparent that the outlines of the individual grains of sand near the inlet (Figures $4 \mathrm{~g}-4 \mathrm{i}$ ) are practically not distinguishable in comparison with top section grains (Figures $4 \mathrm{a}-$ 4c). This visible difference between top and down sections supports the assumption of biofilm colonization with a biomass gradient within the flow cell along the flow direction.

Additional insight on biocolonization has been gained from tracer injections using the Blue Dextran macromolecule, as described in section 2.6. On the basis of the hypothesis that Blue Dextran does not adsorb nor penetrates in biofilm, the effective porosity-as measured with the tracer elution curve-is presumed to decrease with biofilm growth in porous medium. To verify this hypothesis and characterize the longitudinal variation in porous medium colonization by biofilm, tracer experiments were carried out with Blue Dextran at three physical stages of biofilm: (i) without biofilm ( $t=0$ day), (ii) with 17 day biofilm, and (iii) with 29 day biofilm for $Q=1.33 \times 10^{-2} \mathrm{~cm}^{3} \mathrm{~s}^{-1}$ (Figure $5 \mathrm{a}$ ). 

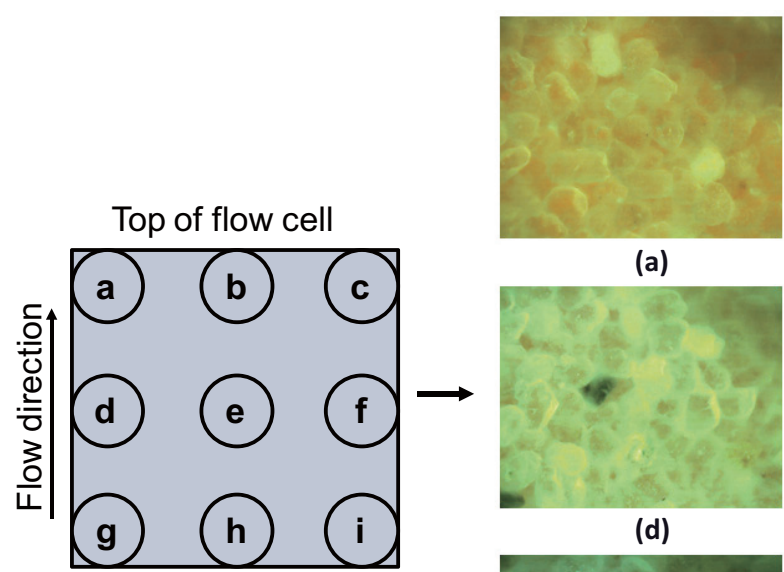

Bottom of flow cell (a)

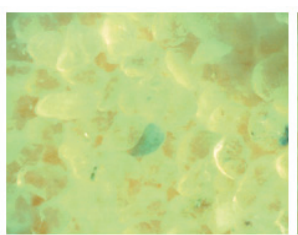

(b)

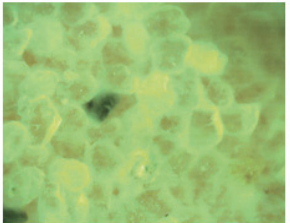

(d)

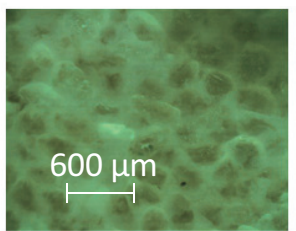

(g)

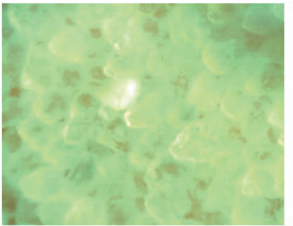

(e)

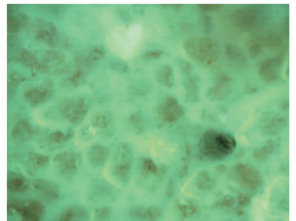

(h)

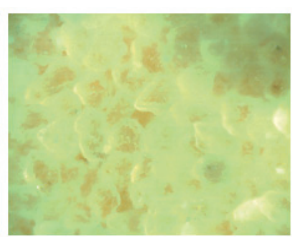

(c)

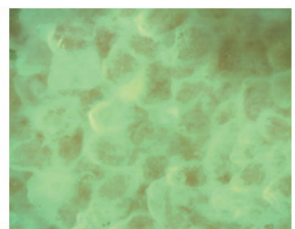

(f)

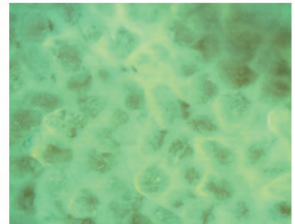

(i)

Figure 4. Observations of biofilm-coated porous medium with microscope AZ100.

Between the inlet of the flow cell and $x=4 \mathrm{~cm}$, results are not physically consistent and cannot be interpreted (data not shown). Calculations lead to values of the effective porosity at $t=0$ day (theoretically equal to the total porosity) higher than the porosity $\varepsilon$ as measured initially. This discrepancy decreases as the cross

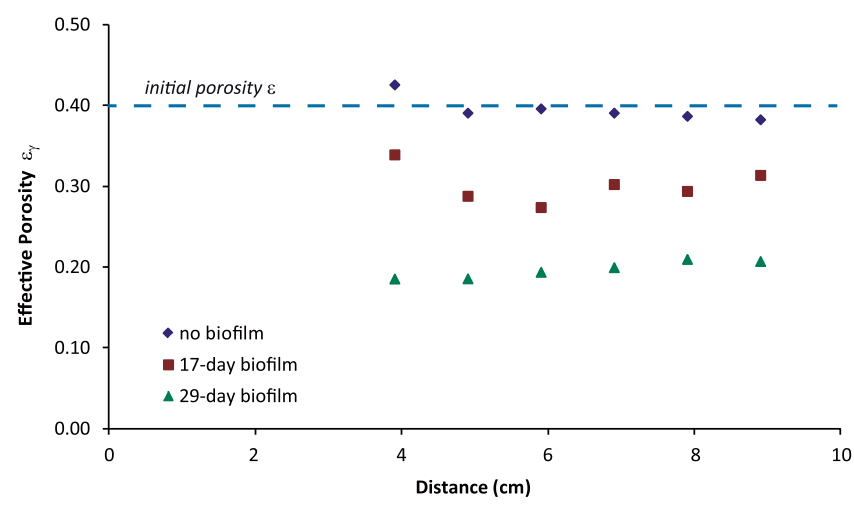

(a)

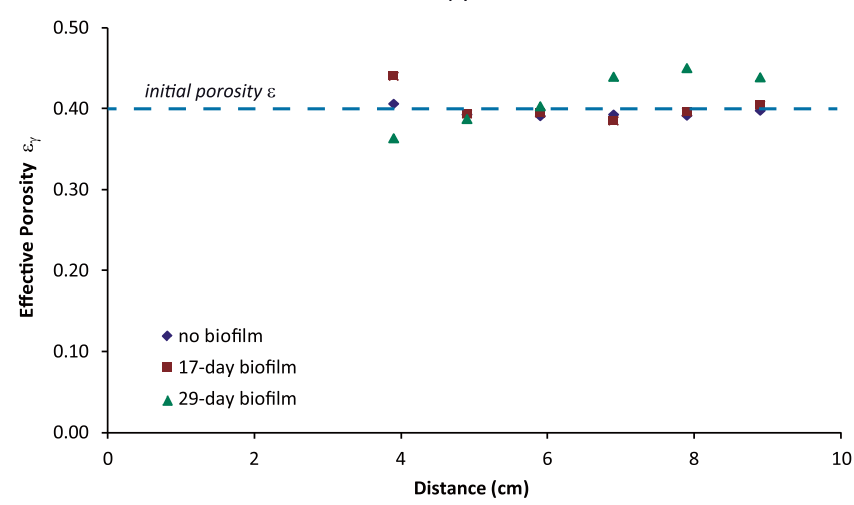

(b)

Figure 5. Evolution of the effective porosity (a) with Blue Dextran and (b) with Brilliant Blue FCF for $Q=1.33 \times 10^{-2} \mathrm{~cm}^{3} \mathrm{~s}^{-1}$. section is further away from the inlet. We think that this experimental error is mainly caused by the injection condition (ramp injection) since the concentration of tracer at the system inlet does not change abruptly from 0 to $C_{\text {max }}$ as in a classical step injection. This change in dye mass between two cross sections leads to a bias in dispersion measurement at early times (see the discussion on a similar issue during interpretation of tracer experiments with continuous mass injection in Mainhagu et al. [2012]). Moreover, this first part of the flow cell is also the most biocolonized with the presence of preferential flow channels that may affect the analysis of breakthrough curves. Thus, the aberrant values of effective porosity within the first $4 \mathrm{~cm}$ could be due to the influence of boundary conditions and to the disturbances caused by strong bioclogging of the porous medium near to the inlet section. In other words, the medium is so heterogeneous that non-Fickian transport prevails and equation (1) cannot be applied [Golfier et al., 2011]. 
Beyond $x=4 \mathrm{~cm}$, the influence of boundary conditions becomes negligible and bioclogging decreases. We recover a Fickian behavior and thus found consistent values for the effective porosity. At $t=0$ day, we obtain as expected a constant value of porosity $(\varepsilon=0.40 \pm 0.02)$ similar to the one measured from volume-balance analysis. Two striking results emerge from measurements at $t=17$ and 29 days (Figure $5 \mathrm{a}$ ). First, for both stages of biofilm maturation, curve analysis indicates a globally constant value of $\varepsilon_{\gamma}(x)$. This plateau suggests a relative homogenous biofilm colonization of porous medium along the flow direction. Obviously, these measurements give no indication about bacterial distribution in the transverse section and preferential flow path may still exist. This finding is also consistent with direct observations with the CCD camera, suggesting a biomass gradient only in the first centimeters (Figure 3). The second finding is that the average value of effective porosity decreases with biofilm growth, which is in agreement with our hypotheses. This behavior suggests a significant biocolonization of the system during the 29 day experiment. Indeed, average biovolume values calculated from breakthrough curves lead to $\varepsilon_{\omega}=0.11$ (i.e., $\varepsilon_{\gamma}=0.29$ or $28 \%$ of initial pore volume colonized) and $\varepsilon_{\omega}=0.2$ (i.e., $\varepsilon_{\gamma}=0.2$ or $50 \%$ of initial pore volume) at day 17 and day 29 respectively. So high values are rarely reached in experimental studies of solute transport in biofilm-coated porous media, except in Seifert and Engesgaard [2012].

In order to support these results and the validity of our analysis, the same methodology has been applied to breakthrough curves of a tracer of low molecular weight, herein Brilliant Blue FCF (Figure 5b), for the same flow rate. Due to propagation and diffusion of this tracer within the biofilm, the three measures yield the same value-corresponding to the initial porosity $\varepsilon$-regardless of the biofilm thickness and this result is consistent with the condition of local mass equilibrium [e.g., Golfier et al., 2009]. Indeed, when the characteristic time for transport in the fluid phase $t_{\gamma}$ is of the same order of magnitude as (or higher than) the characteristic time for diffusion within the biofilm $t_{\omega}$, small macroscale concentration gradients only exist in both phases. The total pore volume participates in flow and a single balance equation (and a single concentration) can be used for describing solute transport. A quick estimate of characteristic time scales confirms this assumption since we have:

$$
t_{\gamma}=\frac{l_{\gamma}}{v_{\gamma}} \sim 2 \mathrm{~s} ; t_{\omega}=\frac{l_{\omega}^{2}}{D_{\text {Blue }}} \sim 0.3 \mathrm{~s}
$$

with $l_{\gamma}$ the characteristic length scale for the fluid phase (about $100 \mu \mathrm{m}$, estimate based on the grain size), $I_{\omega}$ the characteristic length scale for the biofilm phase (between 5 and $10 \mu \mathrm{m}$ assuming a uniform coating of the grains), $v_{\gamma}$ the average pore water velocity (about $5 \times 10^{-3} \mathrm{~cm} \mathrm{~s}^{-1}$ ), and $D_{B l u e}=6 \times 10^{-6} \mathrm{~cm}^{2} \mathrm{~s}^{-1}$.

Comparison with Figure 5 a confirms that variations in effective porosity values in the presence of Blue Dextran are not due to an experimental artifact and justify the validity of our experimental measurement method.

In conclusion, the Blue Dextran method we developed here appears suitable for porous media experiments since it gives access to spatial evolution of biofilm. The current measurements should lead to a better interpretation of coupling effects between microbial growth and solute transport.

\subsection{Breakthrough Elution Curves and Dispersivity Measurement 3.2.1. Influence of Biofilm on Flow Path}

Two-dimensional distributions of the Brilliant Blue FCF concentration were imaged at $t=0,17$, and 29 days. Representative concentration fields of each tracer injection experiments are represented in Figure 6 at the two flow rates for the reduced time $t_{0.5}^{*}$, i.e., after 0.5 pore volume of dye solution was injected.

In the absence of biofilm and for both the injection rates, the packed porous material is homogeneous and we recover a classical hydrodynamic behavior close to plug flow. The front of solute plume remains globally planar even if some slight effects of injection port position and walls appear. As mentioned previously, these experimental flaws are probably caused by the injection method. Indeed, there may be a default of mixing in the reservoir at the entrance of porous medium. This default of mixing may then induce heterogeneities in solute initial concentration at the entrance of porous medium.

As biofilm grows, heterogeneities of porosity and hydraulic conductivity appear and start to impact the propagation of the plume. For the lowest flow rate $\left(Q=1.33 \times 10^{-2} \mathrm{~cm}^{3} \mathrm{~s}^{-1}\right)$, after 17 days of growth, solute concentration is mostly localized in the center of the flow cell. Low concentrations of solute appear also 


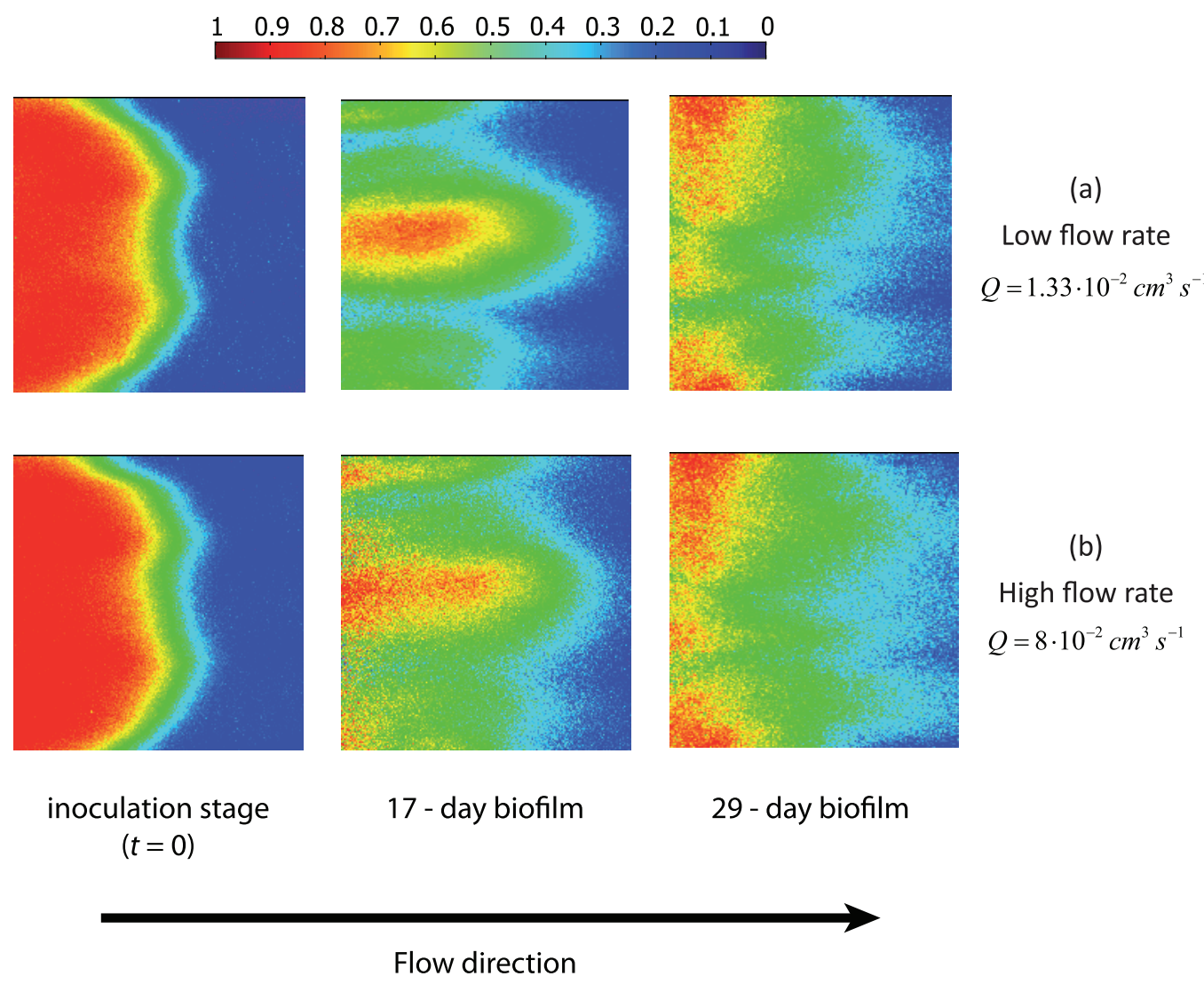

Figure 6. Plume of Brilliant Blue FCF at (a) $Q=1.33 \times 10^{-2} \mathrm{~cm}^{3} \mathrm{~s}^{-1}$ and (b) $Q=8 \times 10^{-2} \mathrm{~cm}^{3} \mathrm{~s}^{-1}$. Images show the solute plume arriving at the middle of the measurement zone. From left to right, the first column shows images of solute plume in the flow cell without biofilm, the second column presents images of solute plume for a17 day biofilm, and the last column for a 29 day biofilm.

along flow cell walls. These observations suggest the appearance of preferential flow paths and thus reveal zones of high concentration of biomass. This phenomenon could be due to heterogeneous clogging of the section of entrance and/or steel sieve (which separates porous matrix and liquid in the reservoir) as previously shown on Figure 3c, which shows the evolution of biofilm in porous medium at day 17. Indeed, a cross analysis of Figures $3 c$ and 6 a clearly gives evidence of two zones of high bioclogging between channels of concentration. After 29 days, on the contrary, heterogeneities in solute concentrations are still noticeable but the path of solute is completely different than for the 17 day mature biofilm. A more homogeneous propagation of solute concentration is observed with peaks appearing near flow cell walls rather than in the central longitudinal section. By analogy with previous observations, we interpret this new spatial distribution of dye concentration as a modification in clogging of entrance section between $t=17$ and $t=29$ days. This behavior is a feature of growth of biotic or nonbiotic aggregates in porous media (e.g., same trend is usually encountered in precipitation processes). Indeed, the development of initial zones of thick biofilm leads to formation of preferential flow paths (behavior at day 17). After 17 days, nutritive solution obviously follows the same preferential flow paths than the tracer dye. This favors the bioclogging of sections initially less colonized whereas the biofilm in sections previously clogged remains constant or decays due to the lack of available nutrients. Ultimately, this competition leads to a progressive homogenization of the system. This result differs from that of Kildsgaard and Engesgaard [2001, 2002] that reported a cyclic bioclogging of a porous medium. This is probably due to sloughing effects which are negligible in our case.

At first sight, results shown in Figures $5 \mathrm{~b}$ and $6 \mathrm{a}$ could appear to be contradictory. Actually, these figures characterize two distinct behaviors. The flow cell can be seen as a two-zone medium: a first region, close to the inlet, highly colonized and strongly heterogeneous and a second region where biofilm growth is quite uniform and Fickian transport regime is recovered. Figure $5 \mathrm{~b}$ characterizes the solute transport within the porous medium between two consecutive cross sections located within the "homogeneous zone" while Figure 6 a shows the solute front after it has flowed through the first half of the flow cell (including the highly 
colonized zone). In other words, this fingering effect, as we can see in Figure 6a, appears during the first centimeters of the plume migration and characterizes the impact of the "heterogeneous zone" on solute transport. Once formed, these fingering remain and are moved through the bed.

At high flow rate $\left(Q=8 \times 10^{-2} \mathrm{~cm}^{3} \mathrm{~s}^{-1}\right.$, Figure $\left.6 \mathrm{~b}\right)$, the same behavior is generally observed for both the 17 and 29 day mature biofilms. However, the spatial distribution of solute concentration is found to be more homogeneous at this flow velocity. This suggests potential slight shearing or erosion of biofilm in highly clogged zones for this flow rate. In any case, if erosion occurs, detachment rate should be a priori quite low since, between two steps of tracer injection, nutrients and washing solutions are circulated through the flow cell at this velocity in order to accustom biofilm to high shear stress.

\subsubsection{Dispersivity Measurement}

Breakthrough elution curves at $x=9 \mathrm{~cm}$ are reported in Figure 7. These results are expressed as reduced concentrations $\bar{c}^{*}$ versus time $t$. Here the concentration $\bar{c}^{*}$ is defined by

$$
\bar{c}^{*}=\frac{\bar{c}-\bar{c}_{\min }}{\bar{c}_{\max }-\bar{c}_{\min }}
$$

where $\bar{c}$ is the averaged-cross-section concentration and $\bar{c}_{\min }$ and $\bar{c}_{\text {max }}$ are, respectively, the average minimal and maximal experimental concentrations of solute in the section of interest.

The analysis of Figure 7 corroborates the influence of biofilm growth on dispersive properties of porous medium, as observed by several researchers both experimentally [Taylor and Jaffé, 1990b; Bielefeldt et al., 2002b] and theoretically [Golfier et al., 2009; Wood et al., 2011]. Indeed, longitudinal dispersion is found to increase with increasing biomass development. Similar behavior is obtained for the two flow rates. The jump of concentration and the slope variation for the lowest flow rate at $t=29$ days (Figure $7 a$, green curve) is probably due to a variation of the flow rate during the tracer injection. To quantify the extent of this phenomenon, longitudinal dispersion has been estimated from measured breakthrough curves using a 1-D convection-dispersion model. Simulations are performed over a domain of $8 \mathrm{~cm}$ length. Due to uncertainty around the physical boundary condition at the inlet, the domain size has been shortened by $2 \mathrm{~cm}$. Instead, experimental breakthrough curves at $x=x_{0}=2 \mathrm{~cm}$ have been introduced into the model using a time-evolving concentration profile $c_{i n j}(t)$ as artificial boundary condition. In this way, we discard the impact of the inlet condition and only measure the changes in dispersion associated to biomass growth in porous media. An example of such time-evolving conditions is shown in Figure 8. Governing equation for solute transport and associated initial and boundary conditions are detailed below:

$$
\begin{gathered}
\varepsilon \frac{\partial \bar{c}}{\partial t}+v \frac{\partial \bar{c}}{\partial x}=\varepsilon D_{L} \frac{\partial^{2} \bar{c}}{\partial x^{2}} \\
\bar{c}(x, 0)=0 \\
\frac{\partial \bar{c}}{\partial x}(L, t)=0 \\
\bar{c}\left(x_{0}, t\right)=c_{i n j}(t)
\end{gathered}
$$

where $\varepsilon=\varepsilon_{\gamma}+\varepsilon_{\omega}$ represents the initial porosity and $v$ is the average Darcy velocity. It is worthwhile to mention that Fickian transport is considered here. This assumption of local mass equilibrium (no mass transfer limitation between fluid and biofilm phases as in a mobile-immobile model for instance) is usually valid for biofilm-coated porous media at low flow rates [Golfier et al., 2009] and may be arguable here. Anyway, we will adopt this approximation in the present work since we will focus only on the determination of the experimental value of $D_{L}$. Note that if the local mass equilibrium condition is verified, the breakthrough should occur in the same time for the three elution curves (for the same flow rate). The discrepancy observed in Figure $7 \mathrm{~b}$ is mainly due to the time-evolving injection condition (caused by the ramp effect at the inlet), which vary between 17 and 29 days. Extensive comparison with numerical simulations and further investigation about the Fickian/non-Fickian behavior of solute transport in the presence of biofilm will be discussed in a following paper. One-dimensional simulations were computed with COMSOL Multiphysics ${ }^{\circledR}$. Numerical parameters are fitted by comparison of the predicted breakthrough curves with observed data at $x=9 \mathrm{~cm}$. Note that these adjustments were conducted manually, without using optimization methods (e.g., linear regression method). 


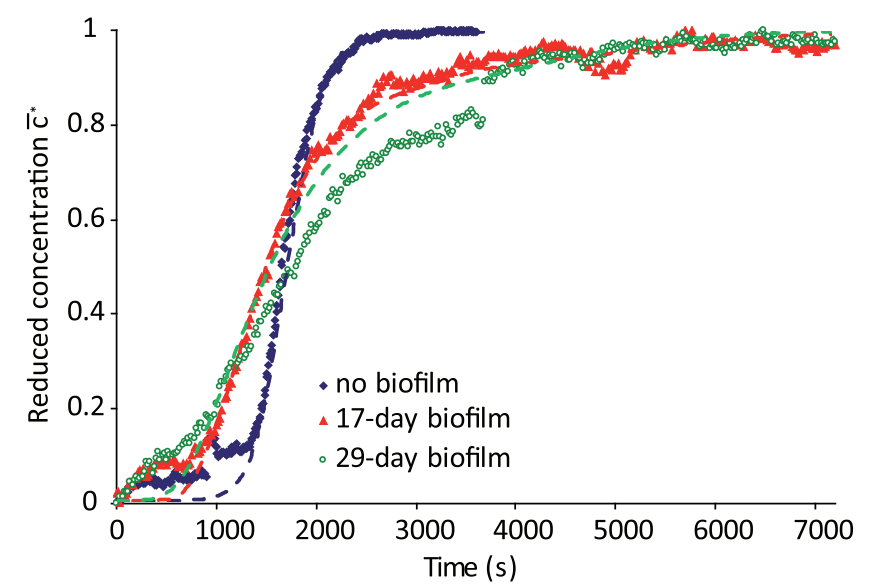

(a)

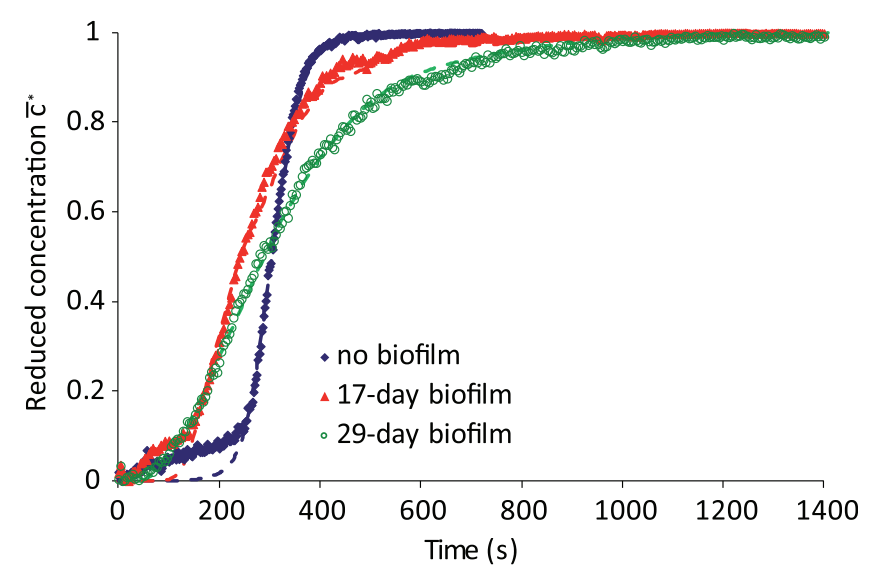

(b)

Figure 7. Brilliant Blue FCF breakthrough curves at $x=9 \mathrm{~cm}$ for different biofilm maturation stages at (a) $Q=1.33 \times 10^{-2} \mathrm{~cm}^{3} / \mathrm{s}$ and (b) $\mathrm{Q}=8 \times 10^{-2} \mathrm{~cm}^{3} / \mathrm{s}$. Numerical simulations fitted with experimental results are represented in dashed lines.
Longitudinal dispersion values determined from numerical simulations are collected in Table 1. In the purpose of exhibiting physical parameters pertinent to this study, injection tests can be also reported as a function of Péclet numbers $P e$, defined as

$$
P e=\frac{v_{\gamma} I_{\text {pore }}}{D_{\text {Blue }}}
$$

Using the molecular diffusion coefficient value of the dye, $D_{\text {Blue }}=6 \times 10^{-6} \mathrm{~cm}^{2} \mathrm{~s}^{-1}$, and the average diameter of the grains as pore-scale characteristic length $\left(I_{\text {pore }}=2.97 \times 10^{-2} \mathrm{~cm}\right)$, the corresponding Péclet values are given in Table 1.

Changes in dispersion are plotted in Figure 9 as a function of biovolume and flow injection rate. First, we notice an increase of dispersion with biovolume, at a given flow rate. In a similar way, at constant effective porosity, the classical dependence of dispersion with pore water velocity is observed. As biofilm grows, it coats more and more the solid carrier leading to grains thicker and hence, an increase of pore water velocity and medium tortuosity. This increase of tortuosity and local velocity field results in increase of dispersion at the macroscopic scale. The discrepancy observed after 29 days is significant and corresponds to about an order of magnitude for a reduction of pore volume by a factor 2 . Results can be more easily interpreted in terms of dispersivity. Following Bear [1972] and assuming molecular diffusion negligible, longitudinal dispersion can be expressed in a general way as

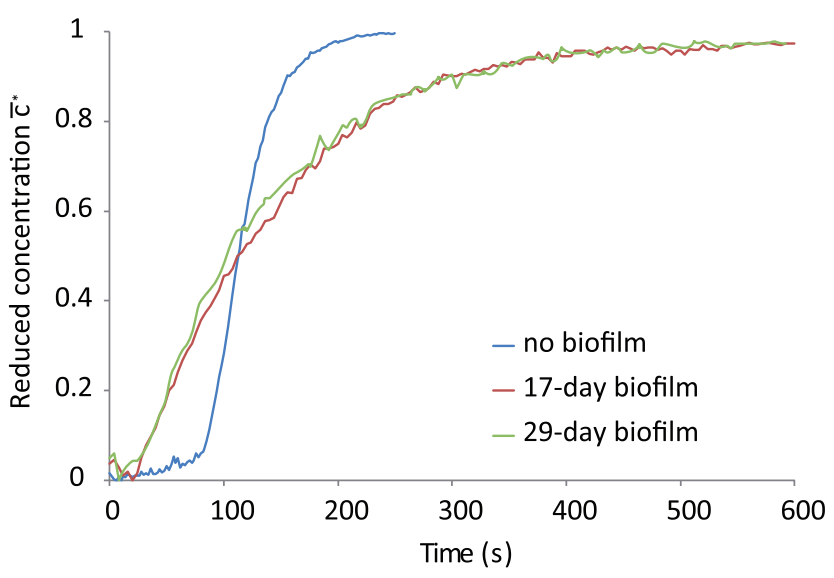

Figure 8. Example of time-evolving boundary conditions measured experimentally at $x=2 \mathrm{~cm}$ and $\mathrm{Q}=8 \times 10^{-2} \mathrm{~cm}^{3} / \mathrm{s}$ for $t=0,17$, and 29 days.

$$
D_{L}^{*}=\frac{D_{L}}{D_{\text {Blue }}}=\alpha_{L}^{*} P e^{n}
$$

with $D_{L}^{*}$ and $\alpha_{L}^{*}$, respectively, the reduced longitudinal dispersion and dispersivity and $n$ the Péclet exponent ( $n=2$ for the Taylor dispersion case). Calculations of $\alpha_{L}^{*}$ and $n$ are reported in Table 1.

From our computations, the Péclet number dependence obtained for the flow cell system yields a quasiconstant value of $n \approx 0.85$, regardless the biofilm thickness. Only the dispersivity varies. This exponent value, close to 1 , indicates a well-packed bed system close a real disordered porous 
Table 1. Experimental Values of Longitudinal Dispersion and Dispersivity Measured at Different Stages of the Biofilm Maturity

\begin{tabular}{llclcc} 
Biofilm Maturation & $\begin{array}{l}\text { Flow } \\
\text { Rate }\end{array}$ & $\begin{array}{c}\text { Péclet } \\
\text { Number }\end{array}$ & $\begin{array}{c}D_{L} \\
\left(\mathrm{~cm}^{2} \mathrm{~s}^{-1}\right)\end{array}$ & $\alpha_{L}^{*}$ & $n$ \\
\hline Without biofilm $(t=0)$ & Low & 26 & $2 \times 10^{-4}$ & 1.95 & 0.87 \\
& High & 165 & $10^{-3}$ & & \\
\multirow{3}{*}{17 day biofilm } & Low & 40 & $5 \times 10^{-4}$ & 3.92 & 0.83 \\
\multirow{2}{*}{29 day biofilm } & High & 213 & $2 \times 10^{-3}$ & & \\
& Low & 51 & $2 \times 10^{-3}$ & 10.4 & 0.88 \\
& High & 316 & $10^{-2}$ & & \\
\hline
\end{tabular}

medium. Variation of $\alpha_{L}^{*}$ with biofilm growth suggests a uniform coating of grains by biological material without abrupt change of pore interconnectivity (quasi-constant value of $n$ ). Calculation of dispersivities using nonreduced values can be more easily interpreted and leads to an increase from $8 \times 10^{-2}$ to $8.5 \times 10^{-1} \mathrm{~cm}$ over 29 days (using $n=1$ for sake of simplicity). The initial value, of the order of the grain size, is physically consistent with a lab-scale system of homogeneous packed bed. The change in dispersivity observed after 17 and 29 days characterizes the occurrence and development of biofilm-induced heterogeneities. Sharp et al. [1999] have reported dispersivity increase from 35 to $288 \%$ due to formation of Pseudomonas aeruginosa biofilm on glass beads. Similar range of dispersivity values were obtained by Seifert and Engesgaard [2007]. Bielefeldt et al. [2002a] have observed the same behavior for multispecies biofilm growth on silica sand with an increase up to 1 order of magnitude. Only Taylor and Jaffé [1990b] have noted more important changes in dispersivity with values higher by a factor 100-1000 relative to the initial value for flow-through experiments in sand column. However, their measures did not agree very well with the theoretical correlation proposed by their model.

To illustrate the interest of this experimental relationship between changes in dispersivity and porosity, a comparison with theoretical predictions of Taylor and Jaffé [1990b] model is provided in Figure 10. As emphasized in section 1, this kind of coupled data are practically nonexistent or at least, porosity estimates are characterized by a significant experimental bias. Taylor and Jaffé's model, based on a cut-and-randomrejoin-type description of the porous medium, is a very rare attempt for predicting influence of biofilm growth on dispersivity and pore volume. Model forecasts are constrained by the value of the pore size distribution index $\lambda$ that characterizes the uniformity of pore throats [Brooks and Corey, 1964]. For comparison purpose with our experimental measures, we used $\lambda=2$, typical value for a porous bed with homogeneous pore sizes (same value is used by Taylor and Jaffé for their experiment in sand column). Figure 10 indicates an excellent agreement between theory and experiment. This agreement should be relativized, yet, due to the uncertainty around the $\lambda$ value. Theoretical prediction with $\lambda=1$ is illustrated in Figure 10 to emphasize this point. It is also worth to be noted that the Taylor and Jaffé model, based on the assumption of a homogeneous biofilm growth, tends to fail for highly heterogeneous material [Vandevivere et al., 1995]. The uniform grain-size distribution of our porous bed could thus explain the good agreement observed with the theory.

For the sake of simplicity, a straightforward function relating dispersivity increase to porosity reduction can be also proposed under the following form:

$$
\frac{\alpha_{L}^{*}}{\alpha_{L}^{*}(t=0)}=k_{\alpha} \cdot\left(\frac{\varepsilon_{\gamma}}{\varepsilon}\right)^{\beta}
$$

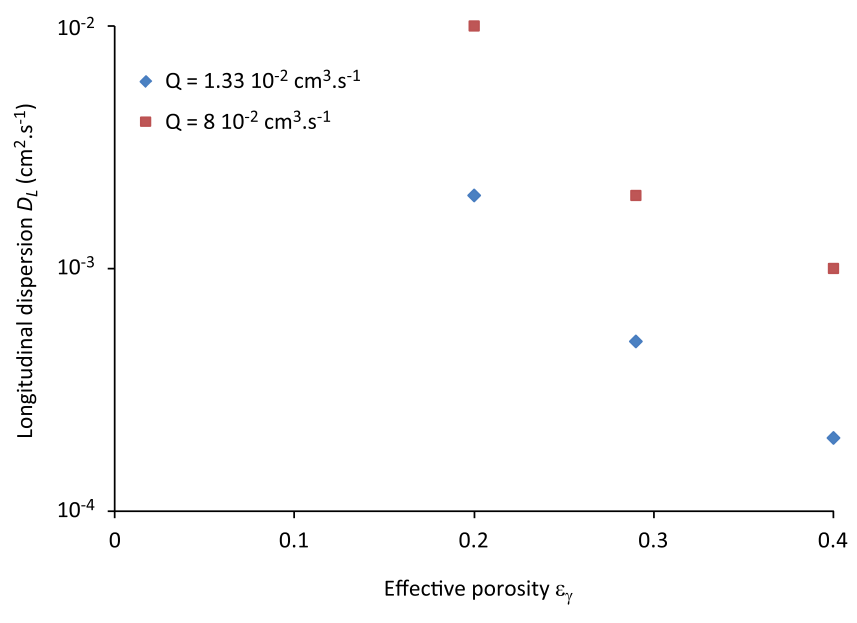

Figure 9. Changes in dispersion as a function of biovolume and flow injection rate.
Constant values $k_{\alpha}=1.0$ and $\beta=-2.3$ are estimated by linear regression. Such simplified expressions can be easily introduced into numerical models of groundwater bioremediation.

\section{Conclusions and Perspectives}

The influence of biofilm growth on flow and transport parameters in porous media has been investigated in this work. Understanding the influence of changes in dispersivity with biofilm growth is a key point for 


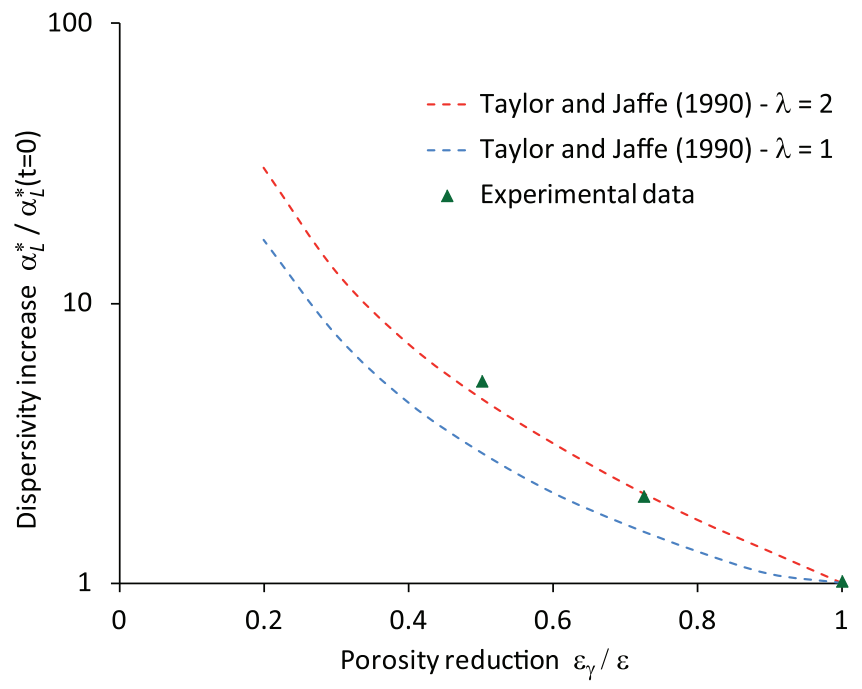

Figure 10. Comparison of dispersivity increase as a function of porosity reduction between experiment and theory [Taylor and Jaffé, 1990a, 1990b, 1990c]. predicting mixing processes in subsurface, which drives biodegradation efficiency. The main outcomes of this study are (i) an original method for measuring in situ the volume fraction of biomass and (ii) in-time observation of the evolving flow paths and tracer plume propagation with biofilm growth.

Biocolonization of the sand-filled flow cell has been monitored during 29 days, and the related changes in effective porosity and longitudinal dispersivity have been measured. As expected, biofilm formation has markedly modified the flow paths and firmly impacted solute mixing within the flow cell. The measurement method of biovo-

lume was based on a similar approach as used in gel-filtration chromatography and revealed a homogeneous and significant (up to $50 \%$ of the initial pore volume) bacterial development along the flow direction. Unlike the results of Rockhold et al. [2005] or Oates et al. [2005] but for lower biofilm volume fractions, our study revealed a real difficulty in the monitoring of solute concentration by optical techniques due to the presence of biomass. A pixel-by-pixel calibration prior to each tracer injection coupled to a postprocessing step was required to image dye concentration fields. From these two data sets, an experimental correlation relating biomass occupation to biofilm-affected dispersivity values was achieved. Such experimental data are very unique and results can be applied as input data of numerical models designed to simulate decontamination scenario and biodegradation efficiency assessment.

Comparison of the dispersivity-porosity relationship with theoretical calculations of Taylor and Jaffé [1990b] has been achieved and excellent agreement was recovered which confirms the reliability of the measurement. Similar comparison with the values of biofilm-affected dispersion predicted by upscaling developments, e.g., from the volume averaging method [Golfier et al., 2009; Orgogozo et al., 2013], could be made and provide significant support for validation of these theoretical works. Impact of biofilm growth on Fickian/non-Fickian behavior of solute transport and development of mass transfer limitations within the biological layer also needs to be questioned. These two points will be the subject of further investigation through a thoroughful comparison with numerical simulations.

\section{Appendix A: Features of the Blue Dextran Dye and Comments on the Biovolume Measurement Method}

The blue Dextran molecule is composed of Dextran, which is a polymer of anhydroglucose, and Reactive Blue 2 dye (approximately $\mathrm{mmol} / \mathrm{g}$ Dextran), which is attached randomly to hydroxyl groups along the Dextran chain. The Blue Dextran is a high molecular weight compound ( $\mathrm{Mw}=2 \times 10^{6} \mathrm{~g} / \mathrm{mol}$ ) classically used in the determination of the void volume for gel-filtration chromatography columns (e.g., in Persson et al. [2006]). Basically, gel-filtration chromatography is a technique which separates on the basis of size, proteins, peptides, and oligonucleotides. The principle is to let molecules move through a bed of porous beads in order to diffuse into the beads. Besides the weight and three-dimensional shape of each kind of molecule, the diffusion degree will be crucial in separation process. Smaller molecules, i.e., molecules whose size is smaller than gel beads, diffuse further into the pores of the beads and thus move through the bed more slowly. On the contrary, larger molecules enter less or not at all inside the gel beads, and thus, move through the bed more quickly. Macromolecules such as Blue Dextran are totally excluded and hence, classically employed to determine the total volume of void within the column. Gels beads may be polysaccharides, which are basic components of biofilm EPS. By analogy, we assumed that Blue Dextran had the same behavior in the presence of both biofilm and gel beads. 
Note that the property of nonadsorption and nondiffusion of Blue Dextran in biofilms due to the anionic nature and high molecular weight of the tracer was already assumed in Jiménez et al. [1988] to evaluate time residence distribution in submerged filters or in Di laconi et al. [2004] but for ex situ determination of biovolume in batch experiments. Moreover, preliminary injection tests coupled with measurements of light absorbance have confirmed that Blue Dextran do not stay permanently in the flow cell after breakthrough and flushing with clean solution.

To the best of our knowledge, Blue Dextran was never used for in situ measurement of biofilm volume fraction in porous media. If the above mentioned research papers support our assumption, several concerns specific to our experimental method could be raised and required to be addressed. First, gel beads used in filtration chromatography possess a well-calibrated microporosity whereas biofilms may possess different level porosity, some of them being potentially greater than the size of Blue Dextran molecules. However, even if such macroporosities exist inside the biofilm growing in the flow cell, the small diffusion coefficient of the Blue Dextran macromolecule $\left(D_{\text {Dextran }}=8.3 \times 10^{-8} \mathrm{~cm}^{2} \mathrm{~s}^{-1}\right.$ from Kommedal et al. [2006]) leads to diffusion characteristic time in microbial phase largely higher than the residence time. Consequently, this point should not have a significant impact on our measurement. Second, Blue Dextran may be biodegraded by bacteria. However, Kommedal et al. [2006] observed biodegradation kinetics of Dextran polymers to be inversely proportional to their molecular weights. Due to the high molecular weight of our dye, we can assume that biodegradation is negligible.

\section{Acknowledgments}

This work was partially supported by the French National Research Agency (ANR) through the MOBIOPOR project, with the reference ANR-10-BLAN-0908, the Carnot Institute funding program (Carnot Institute ICEEL), and the French Scientific Interest Group-Industrial Wasteland (GISFI) program. All data are available on request from the corresponding author.

\section{References}

Baveye, P., P. Vandevivere, B. L. Hoyle, P. C. DeLeo, and D. Sanchez de Lozada (1998), Environmental impact and mechanisms of the biological clogging of saturated soils and aquifer materials, Critical Rev. Environ. Sci. Technol., 28(2), 123-191, doi:10.1080/ 10643389891254197.

Bear, J. (1972), Dynamics of Fluids in Porous Media, Elsevier Sci., N. Y.

Bielefeldt, A. R., C. McEachern, and T. Illangasekare (2002a), Hydrodynamic changes in sand due to biogrowth on naphthalene and decane, J. Environ. Eng., 128(1), 51-59, doi:10.1061/(ASCE)0733-9372(2002)128:1(51).

Bielefeldt, A., T. Illangasekare, M. Uttecht, and R. LaPlante (2002b), Biodegradation of propylene glycol and associated hydrodynamic effects in sand, Water Res., 36, 1707-1714, doi:10.1016/S0043-1354(01)00383-9.

Brooks, R. H., and A. T. Corey (1964), Hydraulic properties of porous media, Hydrol. Pap. 3, Colo. State Univ., Fort Collins.

Charbonneau, A., K. Novakowski, and N. Ross (2006), The effect of a biofilm on solute diffusion in fractured porous media, J. Contam. Hydrol., 85, 212-228, doi:10.1016/j.jconhyd.2006.02.001.

Cunningham, A. B., W. G. Characklis, F. Abedeen, and D. Crawford (1991), Influence of biofilm accumulation on porous media hydrodynamics, Environ. Sci. Technol., 25(7), 1305-1311, doi:10.1021/es00019a013.

Davit, Y., G. Iltis, G. Debenest, S. Veran-Tissoires, D. Wildenschild, M. Gerino, and M. Quintard (2011), Imaging biofilm in porous media using X-ray computed microtomography, J. Microsc., 242, 15-25, doi:10.1111/j.1365-2818.2010.03432.x.

De Muynck, W., N. De Belie, and W. Verstraete (2010), Microbial carbonate precipitation in construction materials, Ecol. Eng., 36, $118-136$.

Di laconi, C., R. Ramadori, A. Lopez, and R. Passino (2004), Preliminary biomass characterization in a sequencing batch biofilm reactor, Ann. Chim., 94, 889-898, doi:10.1002/adic.200490111.

Donlan, R. M., and J. W. Costerton (2002), Biofilms survival mechanisms of clinically relevant microorganisms, Clin. Microbiol. Rev., 15(2), 167-193, doi:10.1128/CMR.15.2.167-193.2002.

Dupin, H., and P. L. McCarty (2000), Impact of colony morphologies and disinfection on biological clogging in porous media, Environ. Sci. Technol., 34(8), 1513-1520, doi:10.1021/es990452f.

Ebigbo, A., R. Helmig, A. B. Cunningham, H. Class, and R. Gerlach (2010), Modelling biofilm growth in the presence of carbon dioxide and water flow in the subsurface, Adv. Water Resour., 33(7), 762-781, doi:10.1016/j.advwatres.2010.04.004.

Engesgaard, P., D. Seifert, and P. Herrera (2006), Bioclogging in porous media: Tracer studies, in Riverbank Filtration Hydrology-Impacts on System Capacity and Water Quality, NATO Sci. Ser. IV Earth Environ. Sci., vol. 60, edited by S. A. Hubbs, chap. 5, pp. 93-118, Springer, Netherlands. doi:10.1007/978-1-4020-3938-6_5.

Golfier, F., B. Wood, L. Orgogozo, M. Quintard, and M. Buès (2009), Biofilms in porous media: Development of macroscopic transport equations via volume averaging with closure for local mass equilibrium conditions, Adv. Water Resour., 32(3), 463-485, doi:10.1016/ j.advwatres.2008.11.012.

Golfier, F., M. Quintard, and B. D. Wood (2011), Comparison of theory and experiment for solute transport in weakly heterogeneous bimodal porous media, Adv. Water Resour., 34(7), 899-914.

Iltis, G. C., R. T. Armstrong, D. P. Jansik, B. D. Wood, and D. Wildenschild (2011), Imaging biofilm architecture within porous media using synchrotron-based X-ray computed microtomography, Water Resour. Res., 47, W02601, doi:10.1029/2010WR009410.

Jiménez, B., A. Noyola, B. Capdeville, M. Roustan, and M. Faup (1988), Design Dextran blue colorant as a reliable tracer in submerged filters, Water Res., 22(10), 1253-1257, doi:10.1016/0043-1354(88)90112-1.

Karrabi, M., P. Séchet, Ch. Morra, A. Cartellier, Ch. Geindreau, and J. M. F. Martins (2011), Investigation of hydrodynamic/biomass growth coupling in a pilot scale granular bioreactor at low pore Reynolds number, Chem. Eng. Sci., 66, 1765-1782, doi:10.1016/ j.ces.2011.01.010.

Kildsgaard, J., and P. Engesgaard (2001), Numerical analysis of biological clogging in two-dimensional sand box experiments, J. Contam. Hydrol., 50, 261-285, doi:10.1016/S0169-7722(01)00109-7.

Kildsgaard, J., and P. Engesgaard (2002), Tracer tests and image analysis of biological clogging in a two-dimensional sandbox experiment, Ground Water Monit. Rem., 22(2), 60-67, doi:10.1111/j.1745-6592.2002.tb00313.x. 
Kim, D.-S., and H. S. Fogler (2000), Biomass evolution in porous media and its effects on permeability under starvation conditions, Biotechnol. Bioeng., 69(1), 47-56, doi:10.1002/(SICI)1097-0290(20000705)69:1<47::AID-BIT6>3.0.CO;2-N.

Kommedal, R., K. Milferstedt, R. Bakke, and E. Morgenroth (2006), Effects of initial molecular weight on removal rate of dextran in biofilms, Water Res., 40(9), 1795-1804, doi:10.1016/j.watres.2006.02.032.

Leis, A. P., S. Schlicher, H. Franke, and M. Strathmann (2005), Optically transparent porous medium for nondestructive studies of microbial biofilm architecture and transport dynamics, Appl. Environ. Microbiol., 71(8), 4801-4808, doi:10.1128/AEM.71.8.4801-4808.2005.

Luckner, L., and H. Reissig (1979), Estimation of the longitudinal dispersion and sorption coefficients in saturated soils by straight-line methods, Hydrol. Sci. Bull., 24(2), 229-238.

MacLeod, F. A., H. M. Lappin-Scott, and J. W. Costerton (1988), Plugging of a model rock system by using starved bacteria, Appl. Environ. Microbiol., 54(6), 1365-1372.

Mainhagu, J., F. Golfier, C. Oltéan, and M. A. Buès (2012), Gravity-driven fingering in fractures: Experimental investigation and dispersion analysis by moment method for a point-source injection, J. Contam. Hydrol., 132, 12-27, doi:10.1016/j.jconhyd.2012.02.004.

Mitchell, R., and Z. Nevo (1964), Effect of bacterial polysaccharide accumulation on infiltration of water through sand, Appl. Microbiol., 12(3), 219-223.

Nambi, I. M., C. J. Werth, R. A. Sanford, and A. J. Valocchi (2003), Pore-scale analysis of anaerobic halorespiring bacterial growth along the transverse mixing zone of an etched silicon pore network, Environ. Sci. Technol., 37, 5617-5624, doi:10.1021/es034271w.

Oates, P. M., C. Castenson, C. F. Harvey, M. Polz, and P. Culligan (2005), Illuminating reactive microbial transport in saturated porous media: Demonstration of a visualization method and conceptual transport model, J. Contam. Hydrol., 77, 233-245, doi:10.1016/ j.jconhyd.2004.12.005.

Orgogozo, L., F. Golfier, M. A. Buès, M. Quintard, and T. Kone (2013), A double-medium theory for groundwater contaminant transport in biofilm-coated porous media, Adv. Water Resour., 62, 266-279.

Paulsen, J. E., E. Oppen, and R. Bakke (1997), Biofilm morphology in porous media, a study with microscopic and image techniques, Water Sci. Technol., 36(1), 1-9, doi:10.1016/S0273-1223(97)00317-X.

Persson, P., P. E. Gustavsson, G. Zacchi, and B. Nilsson (2006), Aspects of estimating parameter dependencies in a detailed chromatography model based on frontal experiments, Process Biochem., 41(8), 1812-1821, doi:10.1016/j.procbio.2006.03.030.

Rockhold, M. L., R. R. Yarwood, M. R. Niemet, P. J. Bottomley, and J. S. Selker (2005), Experimental observations and numerical modeling of coupled microbial and transport processes in variably saturated sand, Vadose Zone J., 4(2), 407-417, doi:10.2136/vzj2004.0087.

Sanchez de Lozada, D., P. Vandevivere, P. Baveye, and S. Zinder (1994), Decrease of the hydraulic conductivity of sand columns by Methanosarcina barkeri, World J. Microbiol. Biotechnol., 10, 325-333.

Seifert, D., and P. Engesgaard (2007), Use of tracer tests to investigate changes in flow and transport properties due to bio-obstruction of porous media, J. Contam. Hydrol., 93, 58-71.

Seifert, D., and P. Engesgaard (2012), Sand box experiments with bioclogging of porous media: Hydraulic conductivity reductions, J. Contam. Hydrol., 136-137, 1-9.

Seymour, J., S. Codd, E. Gjersing, and P. Stewart (2004a), Magnetic resonance microscopy of biofilm structure and impact on transport in a capillary bioreactor, J. Magn. Reson., 167(2), 322-327, doi:10.1016/j.jmr.2004.01.009.

Seymour, J., J. Gage, S. Codd, and R. Guerlach (2004b), Anomalous fluid transport in porous media induced by biofilm growth, Phys. Rev. Lett., 93(19), 198103.1-198103.4, doi:10.1103/PhysRevLett.93.198103.

Seymour, J., J. Gage, S. Codd, and R. Guerlach (2007), Magnetic resonance microscopy of biofouling induced scale dependent transport in porous media, Adv. Water Resour., 30(6-7), 1408-1420, doi:10.1016/j.advwatres.2006.05.029.

Sharp, R. R., A. B. Cunningham, J. Komlos, and J. Billmayer (1999), Observation of thick biofilm accumulation and structure in porous media and corresponding hydrodynamic and mass transfer effects, Water Sci. Technol., 39(7), 195-201, doi:10.1016/S0273-1223(99)00168-7.

Sharp, R. R., P. Stoodley, M. Adgie, R. Gerlach, and A. Cunningham (2005), Visualization and characterization of dynamic patterns of flow, growth and activity of biofilms growing in porous media, Water Sci. Technol., 52(7), 85-90.

Stewart, T. L., and H. S. Fogler (2001), Biomass plug development and propagation in porous media, Biotechnol. Bioeng., 72(3), 353-363, doi:10.1002/1097-0290(20010205)72:3<353::AID-BIT13>3.0.CO;2-U.

Sturman, P. J., P. S. Stewart, A. B. Cunningham, E. J. Bouwer, and J. H. Wolfram (1995), Engineering scale-up of in situ bioremediation processes: A review, J. Contam. Hydrol., 19(3), 171-203, doi:10.1016/0169-7722(95)00017-P.

Taylor, S. W., and P. R. Jaffé (1990a), Biofilm growth and the related changes in the physical properties of a porous medium: 1. Experimental investigations, Water Resour. Res., 26(9), 2153-2159, doi:10.1029/WR026i009p02153.

Taylor, S. W., and P. R. Jaffé (1990b), Biofilm growth and the related changes in the physical properties of a porous medium: 3 . Dispersivity and model Verification, Water Resour. Res., 26(9), 2171-2180, doi:10.1029/WR026i009p02171.

Taylor, S. W., and P. R. Jaffé (1990c), Substrate and biomass transport in a porous medium, Water Resour. Res., 26(9), 2181-2194, doi: 10.1029/WR026i009p02181.

Taylor, S. W., P. C. D. Milly, and P. R. Jaffé (1990), Biofilm growth and the related changes in the physical properties of a porous medium: 2. Permeability, Water Resour. Res., 26(9), 2161-2169, doi:10.1029/WR026i009p02161.

Teal, T. K., D. P. Lies, B. J. Wold, and D. K. Newman (2006), Spatiometabolic stratification of Shewanella oneidensis biofilms, Appl. Environ. Microbiol., 72(11), 7324-7330, doi:10.1128/AEM.01163-06.

Thullner, M., L. Mauclaire, M. H. Schroth, W. Kinzelbach, and J. Zeyer (2002a), Interaction between water flow and spatial distribution of microbial growth in a twodimensional flow field in saturated porous media, J. Contam. Hydrol., 58, 169-189, doi:10.1016/S01697722(02)00033-5.

Thullner, M., J. Zeyer, and W. Kinzelbach (2002b), Influence of microbial growth on hydraulic properties of pore networks, Transp. Porous Media., 49, 99-122, doi:10.1023/A:1016030112089.

Torbati, H. M., R. A. Raiders, E. C. Donaldson, M. J. Mclnerney, G. E. Jenneman, and R. M. Knapp (1986), Biofilm accumulation in sandstone, J. Ind. Microbiol., 227-234.

Vandevivere, P., and P. Baveye (1992a), Effect of bacterial extracellular polymers on the saturated hydraulic conductivity of sand columns, Appl. Environ. Microbiol., 58(5), 1690-1698.

Vandevivere, P., and P. Baveye (1992b), Saturated hydraulic conductivity reduction caused by aerobic bacteria in sand columns, Soil Sci. Soc. Am. J., 56(1), 1-13, doi:10.2136/sssaj1992.03615995005600010001x.

Vandevivere, P., P. Baveye, D. Sanchez de Lozada, and P. DeLeo (1995), Microbial clogging of saturated soils and aquifer materials: evaluation of mathematical models, Water Resour. Res., 31(9), 2173-2180, doi:10.1029/95WR01568.

VanGulck, J. F., and R. K. Rowe (2004), Evolution of clog formation with time in columns permeated with synthetic landfill leachate, J. Contam. Hydrol., 75, 115-139, doi:10.1016/j.jconhyd.2004.06.001. 
Vayenas, D. V., E. Michalopoulou, G. N. Constantinides, S. Pavlou, and A. C. Payatakes (2002), Visualization experiments of biodegradation in porous media and calculation of the biodegradation rate, Adv. Water Res., 25, 203-219, doi:10.1016/S0309-1708(01)00023-9.

Wood, B., F. Golfier, and M. Quintard (2011), Dispersive transport in porous media with biofilm: Local mass equilibrium in simple unit cells, Int. J. Environ. Waste Manage., 7(1-2), 24-48, doi:10.1504/IJEWM.2011.037364.

Yang, S., B. T. Ngwenya, I. B. Butler, H. Kurlanda, and S. C. Elphick (2013), Coupled interactions between metals and bacterial biofilms in porous media: Implications for biofilm stability, fluid flow and metal transport, Chem. Geol., 337-338, 20-29, doi:10.1016/ j.chemgeo.2012.11.005.

Yarwood, R. R., M. L. Rockhold, M. R. Niemet, J. S. Selker, and P. J. Bottomley (2002), Noninvasive quantitative measurement of bacterial growth in porous media under unsaturated-flow conditions, Appl. Environ. Microbiol., 68(7), 3597-3605, doi:10.1128/AEM.68.7.35973605.2002. 